\title{
Application of Hilbert-Huang Transform for Improved Defect Detection in Terahertz NDE of Shuttle Tiles
}

\author{
Robert F. Anastasi ${ }^{1 *}$ and Eric I. Madaras ${ }^{2}$ \\ ${ }^{1}$ U.S. Army Research Laboratory, Vehicle Technology Directorate, AMSRD-ARL-VT-SM, \\ Nondestructive Evaluation Sciences Branch, NASA Langley Research Center, Hampton, VA 23681 \\ ${ }^{2}$ NASA Langley Research Center, Nondestructive Evaluation Sciences Branch, \\ Hampton, VA 23681
}

\begin{abstract}
Terahertz NDE is being examined as a method to inspect the adhesive bond-line of Space Shuttle tiles for defects. Terahertz signals are generated and detected, using optical excitation of biased semiconductors with femtosecond laser pulses. Shuttle tile samples were manufactured with defects that included repair regions unbond regions, and other conditions that occur in Shuttle structures. These samples were inspected with a commercial terahertz NDE system that scanned a tile and generated a data set of RF signals. The signals were post processed to generate C-scan type images that are typically seen in ultrasonic NDE. To improve defect visualization the Hilbert-Huang Transform, a transform that decomposes a signal into oscillating components called intrinsic mode functions, was applied to test signals identified as being in and out of the defect regions and then on a complete data set. As expected with this transform, the results showed that the decomposed low-order modes correspond to signal noise while the high-order modes correspond to low frequency oscillations in the signal and mid-order modes correspond to local signal oscillations. The local oscillations compare well with various reflection interfaces and the defect locations in the original signal.
\end{abstract}

Keywords: Terahertz, Hilbert-Huang Transform, Signal Processing, Nondestructive Evaluation

\section{INTRODUCTION}

The black Space Shuttle tiles that cover the lower surface of the orbiter are known as High-Temperature Reusable Surface Insulation. The tiles are manufactured from silica fibers into a material that is very porous. This material is cut into various shapes and sizes for specific placement on the Shuttle and attached to the Shuttle skin through a bonded strain isolation pad. Integrity of the bond is important and is initially tested by physically pulling on the tile. Terahertz $(\mathrm{THz}) \mathrm{NDE}$ offers a non-contact and high-resolution means of inspecting the tile bond region.

$\mathrm{THz}$ radiation occupies an area of the electromagnetic spectrum between the infrared and microwave bands. Its frequency regime is between $100 \mathrm{GHz}$ and $10 \mathrm{THz}$ with free space wavelength between $30 \mu \mathrm{m}$ and $3 \mathrm{~mm}$. Time-domain signals are generated and detected using optical excitation of biased semiconductors with femtosecond laser pulses. This radiation is able to penetrate and inspect nonconductive material for defects. The energy will propagate through materials and reflect from interfaces that exhibit impedance discontinuities, and can be used in a manner comparable to conventional ultrasonic scanning. Some past uses of $\mathrm{THz}$ have focused on spectroscopic applications such as inspecting the chemical content of packages (Hu and Nuss, 1995), inspection of art work and diagnostics of skin burn depth and

\footnotetext{
*Correspondence: email: robert.f.anastasi@nasa.gov; phone: 757-864-3391; fax: 757-864-4914
} 
severity (Mittlemann, et al., 1999), photonic crystal characterization (Nemec, et al., 2004), and response of THz to human skin (Pickwell, et al., 2004).

For testing purposes, Space Shuttle tiles samples were manufactured with defects. The samples were inspected with a commercial terahertz NDE system that scanned the tile bond-line and generated a data set of RF signals. The signals were post processed to generate $\mathrm{THz} \mathrm{C}$-scan images as are typically seen in ultrasonic NDE. To improve defect visualization, the Hilbert-Huang Transform (HHT) was applied.

The HHT was developed by Huang (Huang, et al. 1998) and used to study and offer physical insight to ocean waves. The motivation for developing this technique was to examine data, physical or numerical, that was non-stationary and non-linear. The ocean wave data was considered highly nonlinear and non-stationary and not suited for Fourier analysis that requires the data to be linear and periodic or stationary. Some other authors used this transform to improve signal analysis by removing signal artifacts. Some example applications include machine tool condition monitoring (Leisk, et al., 2002), civil bridge condition assessement (Addai, 2003), seismic data analysis (Oonincx, 2002 and MagrinChagnolleau, and Baraniuk, 1999), reducing artifacts in electro-gastric signals (Liang et al., 2000 and 2002), and ultrasonic defect mapping (Leisk, 2004). In this paper, the HHT is investigated as a means of improving defect visualization through signal decomposition, but instead of only removing signal noise and signal artifacts, an attempt is made to remove the base signal and retain the buried signal artifacts that may be related to echoes from defects within the sample.

\section{HILBERT-HUANG TRANSFORM}

The central idea of the HHT is the sifting process that decomposes a signal into oscillatory components called intrinsic mode functions (IMF). These functions satisfy the conditions (1) that the number of extrema and the number of zero crossings must be either equal or differ at most by one and (2) that the mean value of the envelope defined by the local maxima and the envelope defined by the local minima is zero. Huang states that the Hilbert transform of these functions are 'well-behaved' and can be used to calculate the instantaneous frequencies.

An IMF is obtained from a given signal, $X(t)$, in three main steps. First, the maxima and minima of the signal are identified and fitted with two smooth cubic-splines, $X_{\text {upper }}(t)$ and $X_{\text {lower }}(t)$. These two curves represent an upper and lower envelope to the signal. In the second step, the mean of the upper and lower envelopes is calculated and subtracted from the original signals to obtain their difference $X_{1}(t)$.

$$
\mathrm{X}_{1}=\mathrm{X}(\mathrm{t})-\left(\mathrm{X}_{\text {upper }}-\mathrm{X}_{\text {lower }}\right) / 2
$$

Step three checks if $\mathrm{X}_{1}$ satisfies the conditions of an IMF. If this result does not satisfy, the conditions then step one and two are repeated using $\mathrm{X}_{1}$. If the result is satisfied then the first IMF, $\mathrm{C}_{1}$, is found and a residual $\mathrm{R}$, is calculated.

$$
\mathrm{R}(\mathrm{t})=\mathrm{X}(\mathrm{t})-\mathrm{C}_{1}(\mathrm{t})
$$

The residual is then used as the new signal and the process is repeated to find subsequent IMF's, $\mathrm{C}_{2}$, $\mathrm{C}_{3}$, etc. The sifting process is stopped when the residual is less then some predetermined value or becomes non-oscillatory thus the number of IMF's depends on the signal and is not a fixed value. When the IMF's and residual are examined the initial IMF, $\mathrm{C}_{1}$, will contain the highest frequency mode of oscillation, subsequent functions will contain decreasing oscillation, and the residual will represent the trend or DC offset of the original signal. The original data can be reconstructed by summing the IMF's and the residual.

\section{SAMPLES}

The Space Shuttle Tile NDE Standard shown in figure 1 consisted of an array of tiles with manufactured defects that included repair regions, insert regions, and other conditions that could occur in Shuttle structures. The NDE Standard 
was scanned with a Terahertz NDE system in an $\mathrm{x}-\mathrm{y}$ array with $2.54 \mathrm{~mm}$ increments. A THz waveform was obtained at each scan location that consisted of 1024 points and time duration of 40 psec. Figure 2 shows a schematic of a tile cross section that consists of a tile, strain isolation pad (SIP) and adhesive area, and aluminum backing. The rays drawn on the schematic highlight the input terahertz signal and echoes from the back of the tile and aluminum.

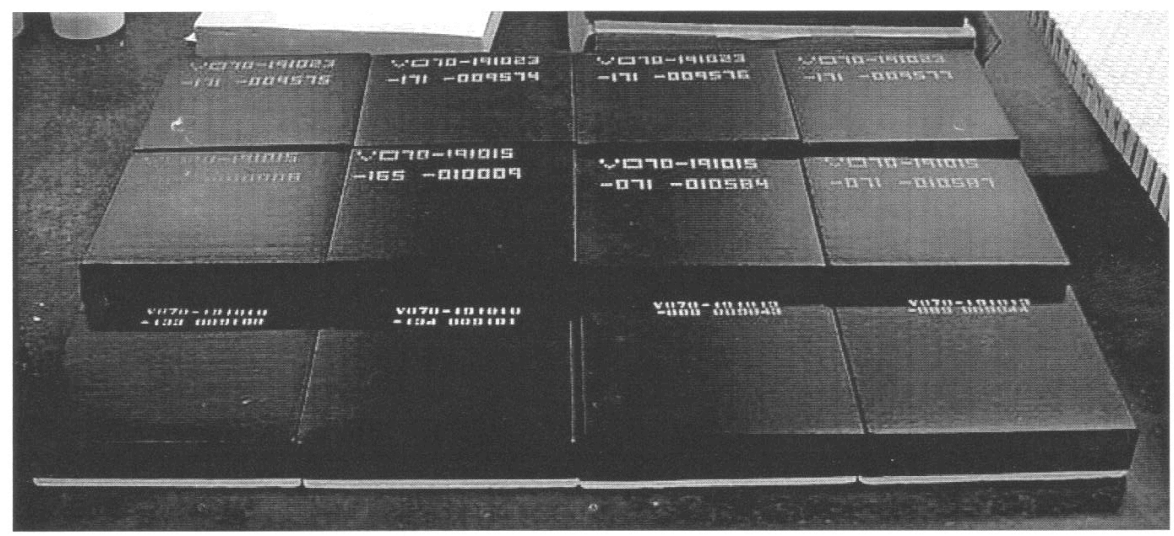

Figure 1. Shuttle Tile NDE Standards

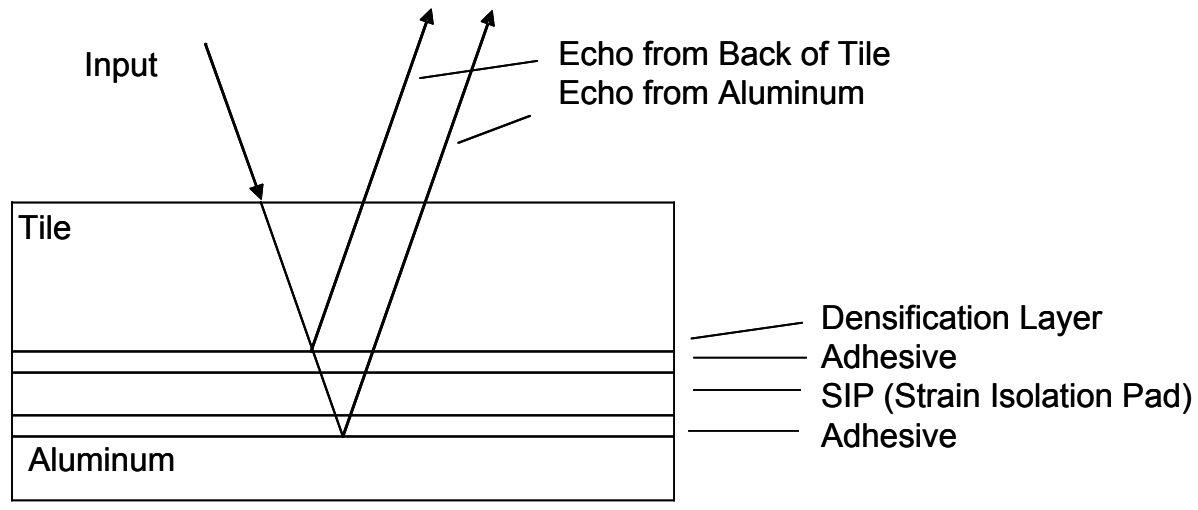

Figure 2. Schematic of a tile cross section is shown with rays drawn for the signal input and echo. The adhesive layers and strain isolation pad is $5-10 \mathrm{~mm}$ thick.

\section{EXPERIMENTS}

The data used in the experiments was from a tile with a triangular insert or defect and was scanned in an $\mathrm{x}-\mathrm{y}$ array of $53 \times 53$ points. A THz C-scan image generated from the scan data is shown in figure 3a. The image was generated using the peak signal at each point. Figure $3 \mathrm{~b}$ schematic of the tile with the defect areas outlined. This image was used to identify signals as being 'in' or 'out' of the defect region. Two test signals were generated by averaging three signals in each of these regions. Signal $S_{\text {out }}$ and $S_{\text {in }}$ shown in figure 4 are the average of three signals 'out' of the defect region and the average of three signals 'in' the defect region respectively. These test signals have a similar shape at the beginning of the signal, deviate in amplitude and time in the SIP area, and deviate in amplitude at the end of the signal. Signal $\mathrm{S}_{\text {in }}$ shows a slight perturbation at about $25 \mathrm{psec}$ that could in part be due to an echo from the defect. The HHT decomposition was applied to these test signals in an attempt amplify their difference. No other form of signal processing was used and only amplitude image comparisons were made to investigate the improvements realized by the HHT decomposition. 


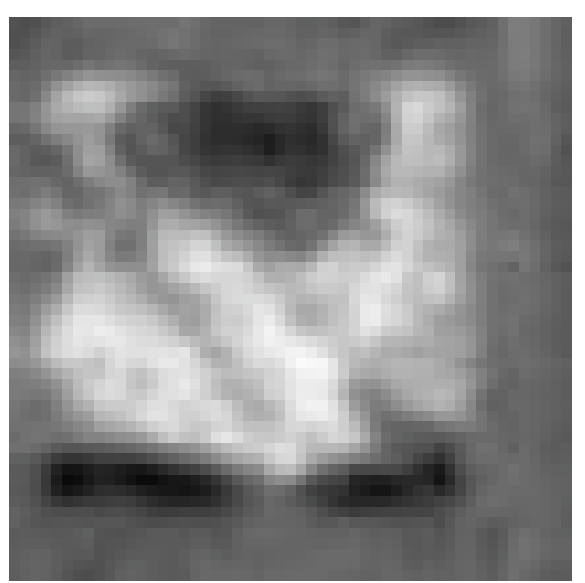

(a)

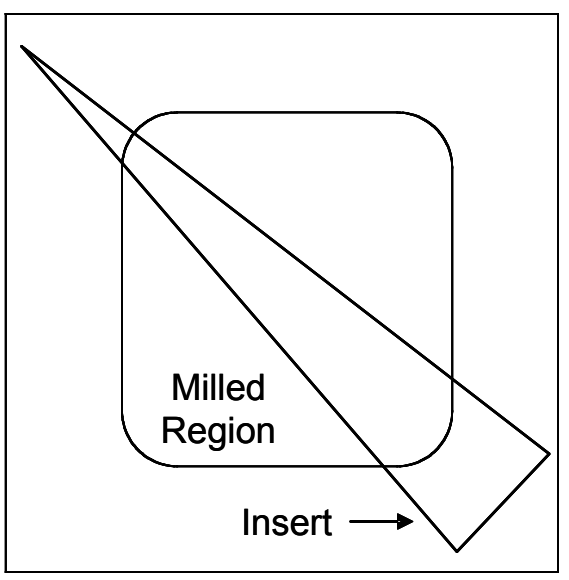

(b)

Figure 3. Tile with triangular insert (a) THz C-scan image of the tile and (b) Schematic showing insert and milled region.

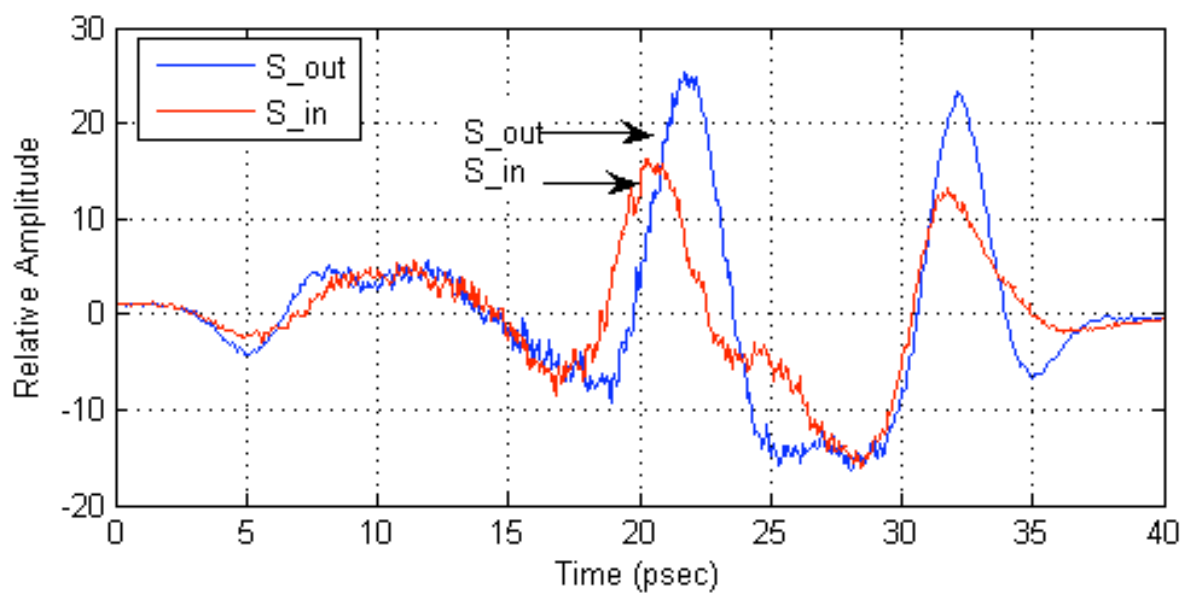

Figure 4. Test signal $\mathrm{S}_{\text {out }}$ is the average of three signals 'out' of the defect region and signal $\mathrm{S}_{\text {in }}$ is the average of three signals 'in' the defect.

\section{RESULT}

An HHT signal decomposition result for the test signal 'in' the defect region is shown in figure 5. This figure shows the signal, the IMF's, and the residual. The initial IMF's are low amplitude and contain the highest frequency mode of oscillation due to noise in the signal, subsequent functions contain decreasing oscillation and amplitudes that increase approximately up to the point of the original signal and then decrease, and the residual is small amplitude and almost a DC type signal.

By examining the IMF amplitude, it can be seen that most of the information is contained in two or three IMF's. By comparing each IMF to the original signal, in an error type of analysis, the IMF that 'best-fit' the original signal was identified. The IMF preceding this 'best-fit' ('best-fit-1') was as also identified. The addition of these two signals comes close to approximating the original signal. This is illustrated in figures 6 and 7 for each test signal. Since the 'best-fit-1' 
has a higher number of oscillator components, this IMF contained some information from the defect. This can be seen in figure 7 in the 'best-fit-1' mode that picks up the signal perturbation at about $25 \mathrm{psec}$.

This process of identifying the best-fit IMF's was applied to a complete data set and $\mathrm{THz}$ C-scan images were generated. Figure 8 shows one time slice of the processed $\mathrm{THz} \mathrm{C}$-scans, (a) shows the processed scan with the identified IMF's added and (b) shows a scan generated with the 'best-fit-1' data. These images can be compared to the original or unprocessed data shown previously in figure 4 . The processed $\mathrm{THz} \mathrm{C}$-scans appear noisier then the original image and may be in part be due to the point-to-point amplitude variations of the signal and identified IMF. The processed scan in figure $8 \mathrm{a}$ is similar to the original scan except for the graininess while the processed scan in figure $8 \mathrm{~b}$ seems to darken and clarify the boundary between the triangular shaped defect and the background.
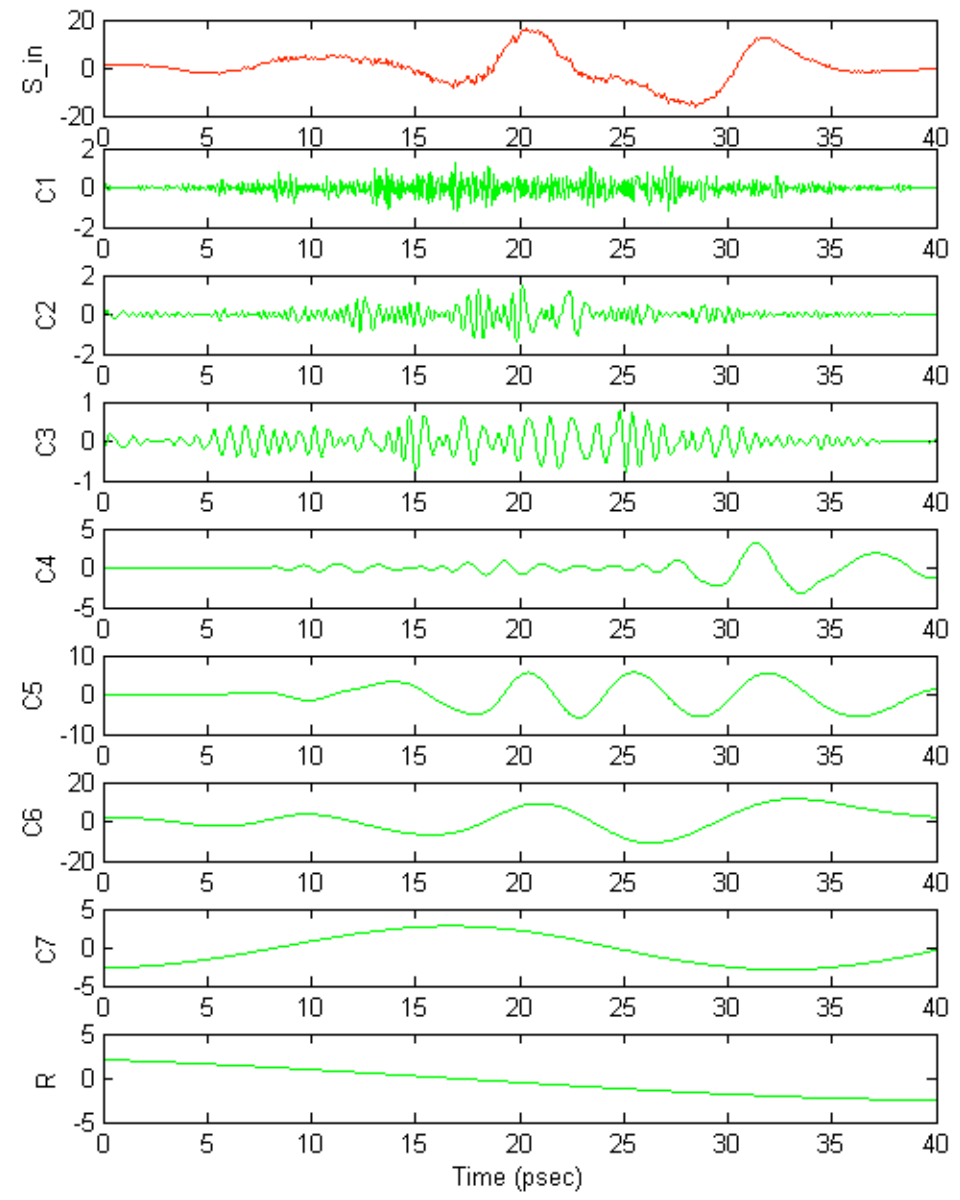

Figure 5. The resulting empirical mode decomposition components for $\mathrm{S}_{\text {in }}$ shown in figure 4, $\mathrm{C} 1$ to $\mathrm{C} 7$ are the decomposed components, and $\mathrm{R}$ is the residual of the decomposition. 


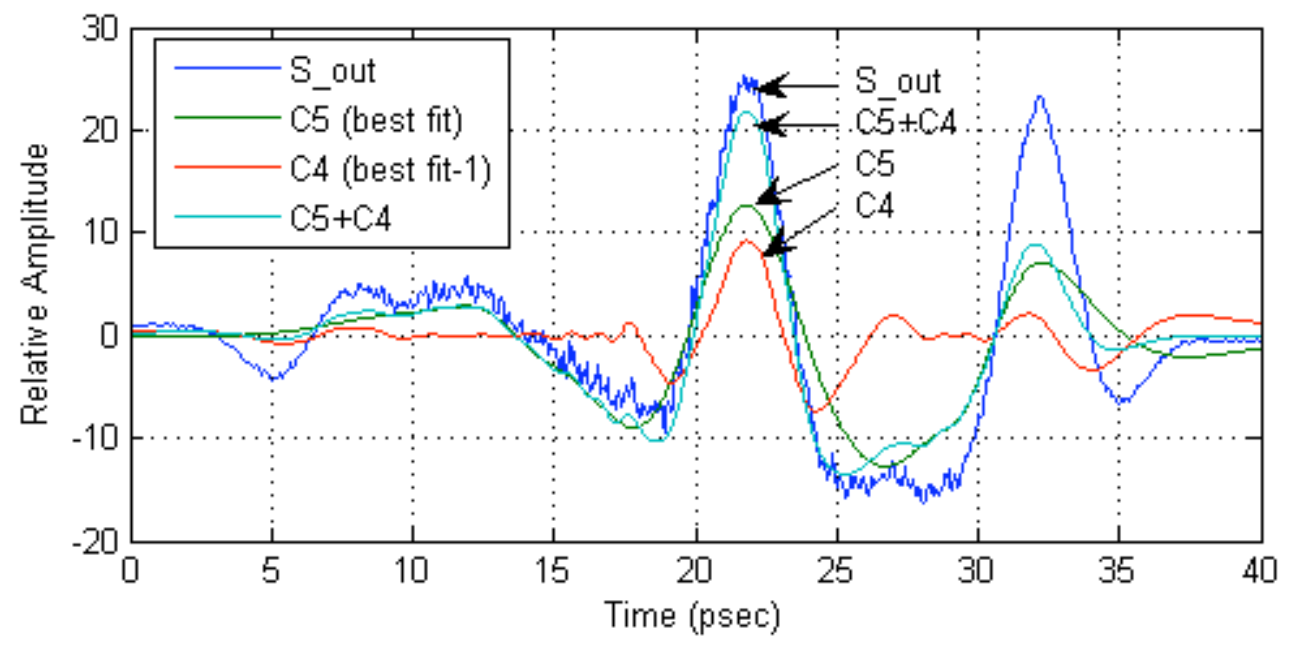

Figure 6. The processing result shows the $\mathrm{S}_{\text {out }}$ the test signal, $\mathrm{C} 5$ the decomposed signal component that best approximates the signal, $\mathrm{C} 4$ the best fit component minus one, and the addition of $\mathrm{C} 5$ and $\mathrm{C} 4$.

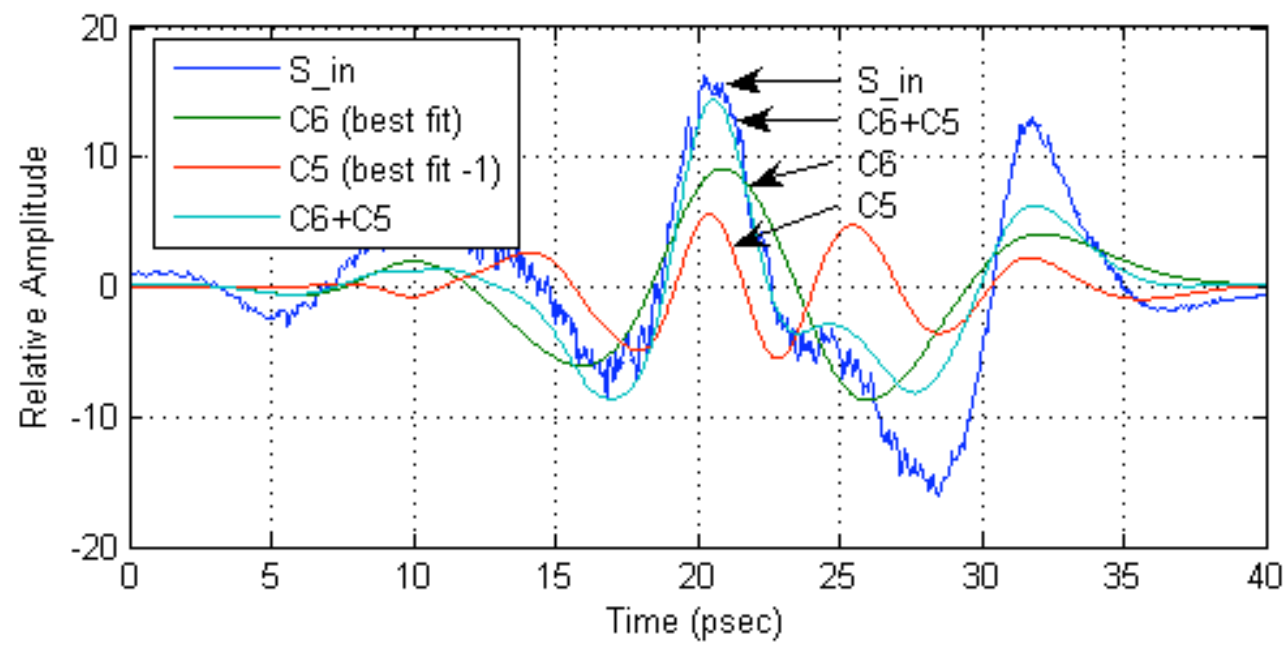

Figure 7. The processing result shows the $\mathrm{S}_{\text {in }}$ the test signal, $\mathrm{C} 6$ the decomposed signal component that best approximates the signal, $\mathrm{C} 5$ the best fit component minus one, and the addition of $\mathrm{C} 6$ and $\mathrm{C} 5$.

\section{CONCLUSIONS}

A data set of RF signals from a terahertz NDE scan of a Space Shuttle NDE standard was examined using the HHT in an attempt to improve visualization of the manufactured defect. The HHT was used to decompose the signal into oscillating components. Specific IMF's were identified based on their relationship to the original signal. One of these identified modes picks up the perturbation that appeared to be from the defect and at the same time de-emphasized the large over powering signal components. This process of identifying specific modes was applied to the complete data. The resulting THZ C-scan improved identification of the boundary between the triangular shaped defect and the background. 


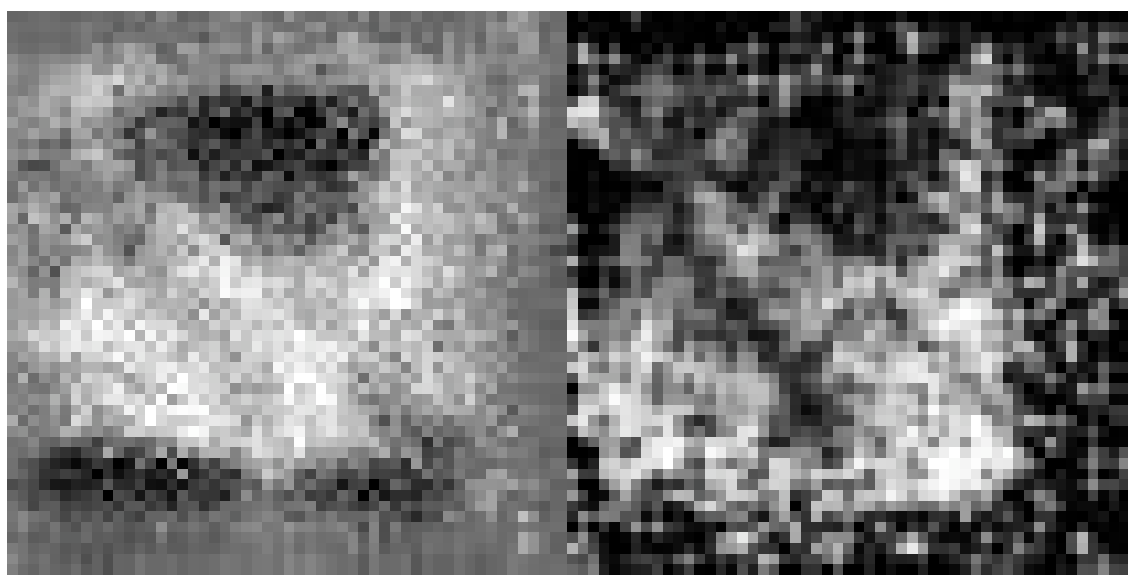

(a)

(b)

Figure 8. Tile with triangular insert is shown (a) after processing individual signals with HHT and adding the IMF with the best fit to the IMF with the best fit minus one, (b) after processing individual signals with HHT and using the IMF with the best fit minus one.

\section{REFERENCES}

Addai, A., "Bridge Sensor Data Analysis Using the Hilbert-Huang Transform," http://www.ce.udel.edu/cibe/reu /REU03/Addai\%20Report.pdf, 2003, accessed October 28, 2004

Haung, N.E., et al., "The Empirical Mode Decomposition and The Hilbert Spectrum for Nonlinear and Non-stationary Time Series Analysis,” Proc. Roy. Soc. London, A454, 903-995, 1998

Hu, B., and Nuss, M.C., "Imaging with Terahertz Waves," Optics Letters, Vol. 20, No. 16, 1995

Leisk, G.G., Miller, E.S., and Murphy, D.J., "Improving Ultrasonic C-Scan Resolution Using The Hilbert-Huang Transform," to be published in Review of Progress in QNDE, Vol. 23, eds. D.O. Thompson and D.E. Chimenti, Plenum Press, New York, 2004

Leisk, Hsu, N.H., and Huang, N.E., "Application of the Hilbert-Huang Transform to Machine Tool Condition/Health Monitoring," Review of Progress in QNDE, Vol. 21, eds. D.O. Thompson and D.E. Chimenti, Plenum Press, New York, 2002

Liang, H., Lin, Z., McCallum, R.W., "Artifact Reduction in Electrogastrogram Based on the Empirical Mode Decomposition Method," Medical and Biological Computing, 38(1), 35-41, 2000

Liang, H. and Lin, Z., "Multiresolution Signal Decomposition and its Application to Electrogastric Signals," Recent Res. Devel. Biomed. Eng. 1, 2002

Magrin-Chagnolleau, I. and Baraniuk, R.G., "Empirical mode decomposition based time-frequency attributes," Proceedings of the 69th SEG Meeting, Houston, Texas, November 1999

Mittlemann, D.M., "Recent Advances in Terahertz Imaging,” Applied Physics B, 68, 1999

Nemec, H., et al., "Time-Domain Terahertz Study of Defect Formation in One-Dimensional Photonic Crystals," Applied Optics, Vol. 43, No. 9, 2004

Oonincx, P.J., "Empirical Mode Decomposition: A New Tool for S-Wave Detection," CWI National Research Institute for Mathematics and Computer Science, Report PNA-RO203, January, 31, 2002

Pickwell, E., et al., "In Vivo Study of Skin Using Pulsed Terahertz Radiation,” Physics in Medicine and Biology, 49, 2004 